\title{
IMPORTÂNCIA DA PRÁTICA CLÍNICA E TECNOLOGIA ALIADAS NA UNIDADE DE TERAPIA INTENSIVA: RELATO DE EXPERIÊNCIA
}

\author{
*Aline Menezes Rossi Tavares \\ Marcilene Rosa Frank"
}

\section{RESUMO}

A prática clínica é de extrema importância no manejo dos usuários admitidos em Unidades de Terapia Intensiva (UTI). No entanto, nem sempre isso é suficiente para se compreender a fundo os agravos em saúde que acometem determinado cliente, tornando-se indispensável o uso de aparatos tecnológicos para a prestação de uma assistência integral e de qualidade. Este estudo tem como objetivo enfatizar a importância e eficácia do uso de aparatos tecnológicos dentro de uma UTI, na qual a utilização da tecnologia foi essencial para obter sucesso em um procedimento de reanimação cardiorrespiratória. Trata-se de um relato de experiência com abordagem descritiva. O relato traz a eficácia dos aparatos tecnológicos que possibilitaram salvar a vida de uma criança, em estado de parada respiratória, ocasião em que só foi possível verificar o motivo de tal acontecimento através do uso de um broncoscópio, identificando que o corpo estranho presente nos brônquios da criança se tratava da metade de um caroço de feijão. Dessa forma, é necessário estimular a reflexão acerca da união dos aspectos clínicos com as ferramentas tecnológicas disponíveis, que são de extrema relevância, pois esta união proporciona um olhar centrado nas necessidades afetadas dos usuários, colaborando com um cuidado cada vez mais sistematizado, humanizado e holístico.

PALAVRAS-CHAVE: Cuidado Intensivo. Equipamentos e Provisões Hospitalares. Ventiladores Mecânicos. Tomografia.

Acadêmica de Enfermagem. Universidade Federal de Mato Grosso - UFMT - Instituto de Ciências Exatas e Naturais - I ICEN. CEP: 78730-302, Rondonópolis - Mato Grosso. Brasil.

*Autor correspondente: alinemenezes123456@gmail.com ORCID ID: 0000-0002-0172-6503; 0000-0002-5259-2138. 


\section{INTRODUÇÃO}

O desenvolvimento e as transformações históricas experimentadas pelas sociedades contam entre as suas causas e efeitos os desenvolvimentos da tecnociência. O uso da informática e de aparelhos modernos, no que tange aos cuidados de enfermagem e saúde, trazem benefícios e rapidez na sistematização influenciado por estas mudanças produzidas no âmbito da tecnologia, o quetem gerado diversas inquietações acerca dos benefícios, riscos e das relações construídas entre trabalhadores, doentes e a utilização de tecnologias como instrumentos imprescindíveis ao cuidado de enfermagem. Principalmente nas unidades de terapia intensiva, o cuidado ao doente crítico envolve a utilização de um arsenal tecnológico específico e que exige, especialmente dos enfermeiros, conhecimentos e habilidades tanto no que se refere à operacionalização de máquinas quanto a sua adequação às necessidades de quem depende dela. ${ }^{1}$

No decorrer da história, é notório que o homem conseguiu aperfeiçoar o seu potencial tecnológico, por meio de objetos simples que foram se transformando em instrumentos diferenciados, evoluindo em complexidade junto com o processo de construção das sociedades. Atualmente, esse processo é nítido em todas as áreas da ciência. ${ }^{2}$ No século $X X$, a ocorrência de duas guerras mundiais gerou uma corrida desenfreada pelo desenvolvimento de tecnologias para a destruição, mas, de forma ambivalente, estas também têm servido para a melhoria da vida das pessoas. A ascensão de máquinas e equipamentos diagnósticos foram os movimentos mais evidentes do avanço tecnológico, no campo terapêutico. Após a Revolução Industrial e a Segunda Guerra Mundial houve mais fomento no crescimento tecnológico e a valorização da ciência, e de forma ambivalente, estas também têm servido para a melhoria da vida das pessoas.,3

Esses avanços da tecnologia também ocorreram na área da saúde, com a introdução da informática e de aparelhos modernos, que trouxeram muitos benefícios e rapidez na batalha contra as doenças. Desenvolveram-se técnicas e aparelhos que facilitam e melhoram as condições de atendimento ao cliente na área da saúde, a exemplo dos precursores de tecnologia no âmbito da saúde que são os instrumentos imprescindíveis ao cuidado.,4 Deve-se salientar, porém, que de nada adianta a posse de tecnologias de alta complexidade, se não for aliada à capacidade humana do profissional que a conduz, no exercício do atendimento com acolhimento. ${ }^{5} \mathrm{~A}$ prática clínica é de extrema importância no manejo dos usuários admitidos em Unidades de Terapia Intensiva, podendo ser compreendida como toda atividade de cuidado, terapêutica, prevenção ou promoção de saúde. Com tal prática, torna-se possível avaliar o estado geral dos usuários, identificar suas necessidades humanas afetadas e planejar ações especificas. ${ }^{2}$

Ciência e tecnologia são instrumentos importantes para a saúde e o tratamento de doenças, assim como para a construção de um momento civilizatório de paz e de vida digna e decente para todos. Dessa forma, a ciência e tecnologia está cada vez mais se inserindo dentro do ambiente hospitalar sendo necessário que as instituições e empresas coloquem a aprendizagem permanente e a produção de novos conhecimentos e tecnologias como centro estratégico de sua sobrevivência e crescimento. ${ }^{3}$ Dessa forma, o presente estudo tem como objetivo enfatizar a importância dos aparatos tecnológicos e a necessidade de integrá-los a prática clínica, principalmente em ambientes como as Unidades de Terapia Intensiva. 


\section{MATERIAL E MÉTODOS}

O estudo trata-se de um relato de experiência feito por uma técnica de enfermagem, acadêmica do $3^{\circ}$ semestre de enfermagem da Universidade Federal de Mato Grosso, observado em uma Unidade de Terapia Intensiva Pediátrica, no município de

\section{RELATO DE EXPERIÊNCIA}

Em um plantão na UTI Pediátrica, foi internada uma criança com 18 meses de vida, proveniente de zona rural do município de Rondonópolis-MT, socorrida pelo Serviço Móvel de Urgência (SAMU) com sintomas de engasgamento e sinais vitais inaudíveis, sendo necessárias manobras de reanimação cardiopulmonar, nas quais se obteve sucesso. No decorrer dos procedimentos de reanimação, estavam presentes quatro técnicos de enfermagem, o enfermeiro responsável pelo setor, um médico intensivista e um fisioterapeuta. Com todos esses procedimentos

\section{DISCUSSÃO}

A elevada complexidade do cuidado da terapia intensiva carece de diversos fatores para o seu desenvolvimento, como uma equipe multiprofissional capacitada e especializada, tecnologia avançada e o uso constante de serviços complementares como exames laboratoriais e de imagem.?

No decorrer do atendimento, a criança teve vários episódios de parada
Rondonópolis-MT, no ano de 2017. Foi feita a descrição de um caso clínico de um paciente pediátrico em parada cardiorrespiratória, sendo indispensável a associação da prática clínica com a tecnologia para realizar um cuidado integral.

e exames, aliados ao cuidado integral de enfermagem, foi possível descobrir a causa de tal engasgamento inicial da criança. O corpo estranho tratava-se da metade de um caroço de feijão em um de seus brônquios, o que impedia a entrada espontânea de oxigênio no pulmão. Após essa descoberta, o caroço foi removido, no entanto, a criança ficou com sequelas neurológicas provenientes da má oxigenação cerebral. $O$ que podemos observar é que a prática clínica aliada a tecnologias como broncoscópio, ventilador mecânico, tomógrafo, entre outros disponíveis no setor, foi de extrema importância.

cardiorrespiratória e, após reanimação, apresentou fibrilação e foi necessário utilizar um desfibrilador para a reanimação. O Desfibrilador Externo Automático (DEA) é um equipamento portátil que possui um sistema computadorizado. É um equipamento de suporte de vida capaz de reverter Fibrilação Ventricular (FV) e a Taquicardia Ventricular (TV), sem pulso para circulação espontânea, 
e que não requer interpretação de traçado eletrocardiográfico, sendo, portanto, de fácil utilização e, por isso, recomendado para leigos e profissionais de saúde, desde que devidamente treinados. ${ }^{8,9}$

Um dos procedimentos comuns realizado dentro de uma UTI é o uso de tubo orotraqueal para o procedimento de intubação e instalação de um suporte ventilatório, através do ventilador mecânico. A ventilação mecânica (VM) é um método de tratamento utilizado para a manutenção da vida em pacientes com insuficiência respiratória que estão nas Unidades de Terapias Intensivas (UTI). É distribuída por meio de uma diversidade de técnicas de maneira parcial, ou completa, chamados modos ventilatórios. ${ }^{10,11}$

Conforme citado no relato, foi utilizado um broncoscópio. Entende-se que a broncoscopia é um método de auxílio diagnóstico e terapêutico. O procedimento permite a visualização das vias aéreas, podendo identificar alterações e realizar coleta de materiais. ${ }^{12} \mathrm{~A}$ broncoscopia tem o papel não

\section{CONCLUSÕES}

Através deste estudo, constata-se o quanto é necessário estimular a reflexão acerca da união dos aspectos clínicos com as ferramentas tecnológicas disponíveis, que são de extrema relevância para a equipe de enfermagem, pois esta união proporciona um olhar centrado nas necessidades afetadas dos usuários que, por sua vez, colabora com um cuidado cada vez mais sistematizado, somente de limpeza e eventual desobstrução por retirada das secreções, mas também de diagnóstico, com identificação do agente patogênico. ${ }^{13}$

Também foram utilizados exames de imagematravés deumtomógrafo. ATomografia Computadorizada (TC) é amplamente utilizada por sua maior disponibilidade, menor tempo de realização, além de possibilitar uma avaliação satisfatória do encéfalo. Através de um exame de Perfusão por TC, podem-se obter os seguintes parâmetros: volume de sangue cerebral e fluxo sanguíneo cerebral. ${ }^{14}$ Sendo indispensável para averiguar a presença de sequelas em nível cerebral.

Mediante o crescente uso de aparatos tecnológicos, dentro do âmbito da saúde, e a comprovação de sua eficácia como neste episódio em uma UTI pediátrica, fica evidente que para o desenvolvimento de uma prática clínica, com uma assistência mais sólida e integral, é de extrema relevância aliar-se as ferramentas tecnológicas.

humanizado e holístico.

Porém, necessário compreender que, além de ter uma reflexão sobre a união dos aspectos clínicos com as ferramentas tecnológicas disponíveis, os profissionais devem ter disponibilidade para se atualizar, participando de cursos de capacitação e de educação permanente para evoluir em conjunto com a tecnologia. 


\title{
IMPORTANCE OF CLINICAL PRACTICE AND TECHNOLOGY ASSOCIATED IN THE INTENSIVE CARE UNIT: EXPERIENCE REPORT
}

\begin{abstract}
Clinical practice is extremely important in the management of users admitted to Intensive Care Units (ICUs). However, this is not always sufficient to fully understand the health problems that affect a particular client, making it essential to use technological devices to provide comprehensive and quality care. This study aims to emphasize the importance and effectiveness of the use of technological devices within an ICU, which the use of technology was essential to achieve success in a cardiorespiratory resuscitation procedure. It is an experience report with a descriptive approach, the report brings the effectiveness of technological devices that made it possible to save the life of a child in a respiratory arrest, where it was only possible to verify the reason for such an event through the use of a bronchoscope, identifying that the foreign body present in the child's bronchi was the size of half bean pit . Thus, it is necessary to stimulate reflection about the union of clinical aspects with the available technological tools, which are extremely relevant, as this union provides a look centered on the affected needs of users, collaborating with an increasingly systematized, humanized and holistic.
\end{abstract}

KEYWORDS: Critical Care. Hospital Equipment and Supplies. Mechanical Ventilators. Tomography.

\section{REFERÊNCIAS}

1. Schwonke CRGB, Filho WD, Filho, Lunardi VL, Santos SSC, Barlem ELD. Perspectivas filosóficas do uso da tecnologia cuidado de enfermagem em terapia intensiva. Rev Bras Enferm. 2011;64(1):189-192.

2. Almeida EF de. Assistência de enfermagem em UTI frente ao uso de tecnologias: Uma Revisão Integrativa. [monografia]. São Luís. Universidade Federal do Maranhão; 2017.

3. Leite CRM, Rosa SSRF. Novas Tecnologias Aplicada à Saúde: Integração de Áreas Transformando a Sociedade. 1. ed. MOSSORÓ: EDUERN, 2017.

4. Barra DCC, Nascimento ERP, Martins JJ, Albuquerque GL, Erdmann AL. Evolução histórica e impacto da tecnologia na área da saúde e da enfermagem. Rev. Eletr. Enf. 2006: 8(3):422-30.

5. Marques IR, Souza AR. Tecnologia e humanização em ambientes intensivos. Rev Bras Enferm. 2010; 63(1): 141-44.

6. Coelho MO, Jorge MSB. Tecnologia das relações como dispositivo do atendimento humanizado na atenção básica à saúde na perspectiva do acesso, do acolhimento e do vínculo. Ciênc. Saúde Colet. 2012;14(1):1523-31.

7. Souza NS, Souza TSB de, Chagas FRC, Silva NF da, Silva SV, Silva CC. Repercussões das tecnologias do cuidar nas unidades de terapia intensiva. Rev Enferm UFPE online. 2018;12(10):2864-72. 
8. Oliveira NS. Efetividade do desfibrilador externo automático no suporte básico e avançado de vida intra-hospitalar. [dissertação de mestrado]. Rio de Janeiro. Universidade Federal do Estado do Rio De Janeiro; 2018.

9. Aehlert B. Emergências em cardiologia: suporte avançado de vida em cardiologia. $3^{\mathrm{a}}$ ed. Texas: Elsevier, 2007.

10. Souza FLS, Costa MLA, Daltro MCSL, Candeira DA. Desmame da ventilação mecânica realizado em unidade de terapia intensiva: uma revisão sistemática. Temas em Saúde. 2019; 19(1):40-60.

11. Santos CR dos, Costa BHS, Dias TKC, Guedes HCS, Paz MSS da, Oliveira TC de, et al. Fatores de risco que favorecem a pneumonia associada à ventilação mecânica: Artigo de Revisão Integrativa. Rev Enferm - UFPE online. 2018;12(12):340115.

12. Mafort TT. O papel da broncoscopia no manejo dos pacientes com doenças pulmonares supurativas. Pulmão RJ 2014;23(3):19-20.

13. Costa Jr. AS, Scordamaglio PR, Suzuki I, Palomino AL, Jacomelli $M$. Indicações, resultados clínicos e complicações de 1.949 broncoscopias flexíveis. Einstein (São Paulo). 2018;16(4).

14. Silva FMS, Oliveira EMF. Comparação dos métodos de imagem (tomografia computadorizada e ressonância magnética) para o diagnóstico de acidente vascular encefálico. Rev Enf Contemporânea. 2017;6(1):81-89. 